18. In this paper Dr. Lennes describes in detail the construction of a set which is non-measurable in the sense of Lebesgue. The general method is the same as that used by Van Vleck (Transactions, volume 9, page 237), but each step is made to depend upon explicitly formulated postulates.

19. In two papers published in the American Journal of Mathematics, volume 33, Dr. Lennes formulated a certain body of theorems on polygons and polyhedrons. With one exception these were confined to figures having a finite number of sides or faces. In the present paper many of the results obtained in these papers are extended to polygons and polyhedrons having an infinite number of sides or faces.

F. N. Cole, Secretary.

\title{
PROOF OF A THEOREM DUE TO PICARD.
}

BY PROFESSOR W. R. LONGLEY.

(Read before the American Mathematical Society, April 27, 1912.)

CoNSIDER the ordinary differential equation of the first order and second degree

$$
A p^{2}+2 B p+C=0 \quad(p=d y / d x),
$$

in which the coefficients are power series in $x$ and $y$ vanishing when $x=y=0$

$$
\begin{gathered}
A=a x+a_{1} y+\cdots, \quad B=b x+b_{1} y+\cdots, \\
C=c x+c_{1} y+\cdots .
\end{gathered}
$$

Picard has proved that in the general case* every integral curve (real curve in the cartesian plane) of equation (1) which comes infinitely near the origin, actually reaches the origin with a determinate tangent. The proof $\dagger$ is based upon the existence of an analytic integral curve passing through the origin, a condition which is satisfied in the general case. But such a curve does not always exist, and the following proof, which

* By the general case it is meant that there exists no particular relation of equality among the coefficients of (2).

† Picard, Comptes Rendus, vol. 120 (1895) p. 524; Math. Annalen, vol. 46 (1895), p. 521; Traité d'Ánalyse, vol. 3, pp. 217-225. 
makes no use of its existence, gives more information concerning the form of the integral curves near the origin.

To examine the behavior of integral curves in the neighborhood of the origin we may limit the coefficients in equation (1) to terms of lowest degree and we suppose that $A, B$, and $C$ contain only first degree terms in $x$ and $y$. Solving equation (1) for $p$ gives

$$
p=\frac{-B+\sqrt{\Delta}}{A},
$$

where $\Delta=B^{2}-A C$. Through every point at which $\Delta$ does not vanish there are two integral curves with distinct tangents depending on the choice of the sign of the radical. Suppose this choice has been made. Then the form of equation (3) shows that the slope depends only on the ratio $y / x$ and hence the slope of an integral curve crossing the line $y=m x$ is the same at every point of the line. From this it follows that it is possible to trace a continuous integral curve by making the radius vector from the origin turn always in the same direction, provided the curve does not reach a point at which $\Delta=0$ where the sign of the radical could be changed.

The form of the integral curves depends on the nature of the discriminant locus, the equation of which is $\Delta=0$, and there are two cases to be considered: (1) when the discriminant locus has a conjugate point at the origin, and (2) when the discriminant locus consists of two distinct branches through the origin.

In the first case $\Delta$ vanishes for $x=y=0$ and is positive for all other values of $x$ and $y$. Hence if an integral curve does not pass through the origin it may be followed by making the radius vector rotate always in the same sense about the origin. It is conceivable that an integral curve might have the form of a spiral approaching the origin asymptotically. To do this the slope must change sign four times during one revolution of the radius vector; twice by passing through zero (at the highest and lowest points) and twice by passing through infinity (at the extreme right and left points). To see that this is impossible we consider equation (3). The lines $A\left(a x+a_{1} y=0\right), B\left(b x+b_{1} y=0\right)$, and $C\left(c x+c_{1} y=0\right)$ pass through the origin and by a rotation of the axes the line $B$ may be taken for the $X$-axis. The line $C$ is the locus of points where the slope of integral curves is zero. Starting at any 
point near the origin on the positive $X$-axis with a definite sign for the radical we may follow an integral curve by making the radius vector rotate always in a counterclockwise direction. If the slope changes sign by passing through zero when the curve crosses the line $C$ above the $X$-axis, then $p$ will not change sign when the same line is crossed below the $X$-axis, because the sign of the radical remains the same while the sign of the quantity $B$ is reversed. Hence the curve can not meet the positive $X$-axis a second time and the spiral is impossible.

In the second case the discriminant locus has two distinct branches passing through the origin. In one of the angles between the branches $\Delta$ is positive and there exist real integral curves, while in the other angle $\Delta$ is negative and there are no real integral curves. By a transformation of coordinates we may take the two branches of the discriminant locus for the coordinate axes so that $\Delta$ is positive in the first and third quadrants and negative in the second and fourth quadrants. Since $\Delta=B^{2}-A C$ is positive in the first quadrant and negative in the second it is evident that the lines $A$ and $C$ must pass through the origin with positive slope, that is, they must lie in the first quadrant. In this case an integral curve can not have the form of a spiral, but it is conceivable that it might oscillate between the coordinate axes an infinite number of times approaching the origin. To see that this is impossible suppose that we choose a definite sign for the radical in equation (3) and begin to follow an integral curve from a point on the positive $X$-axis. The radius vector must rotate in a counterclockwise direction until the $Y$-axis is reached. To follow the curve further the sign of the radical must be changed at this point and the radius vector turned clockwise towards the $X$-axis. Each time the curve traverses the first quadrant it must cross the line $A$ once and the line $C$ once. In crossing the line $C$ with one sign for the radical the slope of the curve will change sign by passing through zero, while with the opposite sign for the radical the slope of the curve will not change sign in crossing $C$. Similarly in crossing the line $A$ with one sign for the radical the slope will change sign by passing through infinity, while with the opposite sign for the radical the slope will not change sign. Hence in starting from the $X$-axis, crossing to the $Y$-axis, and returning to the $X$-axis the slope of an integral curve must change sign twice and only 
twice; once by passing through zero (at a maximum or minimum point) and once by passing through infinity (at an extreme right or extreme left point).

Suppose now an integral curve $\Gamma$ leaves the $X$-axis with positive slope. In order to reach the $Y$-axis the slope must become negative when $\Gamma$ crosses $A$. Then in crossing $C$ (either the first or second time) the slope will change to positive by passing through zero, and with a positive slope $\Gamma$ can not return to the $X$-axis.

Suppose $\Gamma$ leaves the $X$-axis with negative slope and meets $A$ first. In order to reach the $Y$-axis it must cross $A$ the first time without changing to positive slope. After having crossed $C$ twice the curve will return towards $A$ with positive slope and can not again meet $A$ because at the second point of intersection the slope of $\Gamma$ would have to be infinite. Hence $\Gamma$ can not return to the $X$-axis.

Suppose $\Gamma$ leaves the $X$-axis with negative slope and meets $C$ first. (a) If the slope does not change sign then $\Gamma$, after crossing $A$ twice, will return towards $C$ with positive slope and can not meet $C$ again because at a second point of intersection the slope would have to be zero. (b) If the slope changes to positive at the first intersection with $C$ then the curve could not cross $A$ twice since the slope would have to be infinite at one of the points of intersection. Hence $\Gamma$ can not return to the $X$-axis.

The preceding argument establishes the theorem as given by Picard. No mention has been made of the case in which the discriminant locus has a cusp at the origin. If the coefficients in equation (1) are limited to terms of the first degree, a cusp at the origin means that $\Delta$ is a perfect square and the equation can be broken up into linear factors. In this case the spiral is a possible form for integral curves, but this conclusion does not follow when the higher degree terms are present unless the cusp degenerates into two coincident branches.

Sheffield Scientific School, New Haven, Connecticut. 